\title{
From the library
}

Remembrance of things past

His eyes circled by rings he rarely sleeps. At times he wakes up with a start from terrifying dreams, nightmares whose nature Isabelle would not tell us, but they seem as atrocious as those as which he afflicted the Verlaine of London, "the eyes torn out," "the heart filled with rums." Rimbaud is no longer a text at this moment but a body. (Jean-Luc Steinmetz. Arthur Rimbaud. Presence of an Enigma. New York: Welcome Rain Publishing, 2001:374)

\section{Retinal problem associated with vigabatrin}

Vigabatrin is an effective first line drug for the treatment of many seizure disorders. It is an inhibitor of GABA transaminase and thus produces elevated GABA in the brain and perhaps the retina. In adults visual field loss associated with vigabatrin has now been reported in up to $50-70 \%$ of patients. Many neurologists have stopped using vigabatrin. A report from Oman suggests that in children the toxicity is equally devastating. In a series of 21 children using vigabatrin, primarily for the treatment of infantile spasms, 16 of them developed abnormal visual evoked potentials and four developed abnormal retinal findings on ophthalmoscopy. Regrettably, electroretinography and electro-oculography were not performed in these patients. (Archives of Disease in Childhood 2001;85:1-3)

\section{Obesity and age related eye diseases}

Although the precise aetiology of age related macular degeneration is yet unknown some risk factors have been identified. Participants of the Beaver Dam Study at 5 years' follow up demonstrated that there was an increased risk for age related eye disease in women with an increased waist to hip ratio rather than just the body index alone. It is not clear if adiposity is a risk factor on its own or whether it is a confounder of other risk factors for age related ocular disease. (Ophthalmic Epidemiology 2001;8:251-62)

\section{Psoriasis and associated malignancy}

Psoriasis is a relatively common dermatological disorder with occasional ocular manifestations. In a recent cohort study from the University of Pennsylvania it was demonstrated that individuals with severe psoriasis were much more likely to develop a malignancy than those with hypertension. Indeed, the risk of malignancy in the severe psoriasis group approached the risk for that of patients with organ transplants. Most of these cancers were non-melanoma skin cancers and lymphoproliferative malignancies. In less severe forms of psoriasis the risk for malignancy is essentially the same as for the control group with hypertension. (Archives of Dermatology 2001;137:778-83)

Iron deficiency and its effect on cognitive abilities

Iron deficiency anaemia in infants is known to cause developmental problems. In a recent study from Rochester, New York, an older set of children was studied. Among the 5398 children in the sample 3\% were found to be iron deficient. Average maths scores were lower for children with iron deficiency whether they had anaemia or not. Screening for iron deficiency even when no anaemia is apparent may be warranted in children at risk. (Pediatrics 2001;107:1381-6)

\section{Lyme disease prophylaxis}

Lyme disease is recognised with increased frequency in various parts of the world. It is a disorder that occurs after a bite by an infected tick. The disease is characterised by fever, arthralgias, nausea, and skin rashes. Ocular involvement may include iritis and optic neuritis. In a study from New York a single dose of $200 \mathrm{mg}$ doxycycline given within 72 hours after the infected tick bite appeared to prevent the development of Lyme disease. (Nerw England Fournal of Medicine 2001;345:75-84)

\section{More on the risk of air travel}

The risk of thromboembolism during long aeroplane flights has been the subject of numerous recent reports. The LONFLIT study was planned to evaluate the incidence of deep venous thrombosis occurring as the consequence of long aeroplane flights. In this study the average flight duration was 12.4 hours and all subjects flew in economy class. Low risk subjects (who have no cardiovascular disease and used no drugs) had no episodes of deep venous thrombosis, whereas in high risk subjects (primarily patients with cardiovascular disease) 11 of 389 subjects had deep venous thrombosis and six had superficial thromboses. The use of below the knee stockings was also evaluated and the incidence of deep venous thrombosis was 18.75 times lower than for subjects who did not wear stockings. (Angiology 2001;52:369-74)

Oestrogen use and cataract development A number of studies of oestrogen replacement therapy have produced conflicting reports as to whether or not it is associated with decreased risk of cataract formation. The surviving members of the original cohort of the Framingham Heart Study have recently been examined for the absence or presence of lens opacities. In this study the longer duration of post-menopausal oestrogen therapy was inversely associated with the presence of nuclear lens opacities in an age adjusted model. This study along with several others suggests that there is a reduced risk for lens opacities in post-menopausal women taking oestrogen supplements. (Archives of Internal Medicine 2001;161:1448-54)

Visual attention deficits after subcortical vascular lesions

Attention deficits are among the most frequent neuropsychological consequences of brain injury. Among these, visual attention mechanisms are increasingly being defined. In a recent study from Germany 15 patients with acute circumscribed vascular lesions confined to subcortical structures such as the basal ganglia, internal capsule, and thalamus were studied. In this study there was a clear attentional asymmetry in patients with right hemispheric lesions. Seven out of the eight patients with right sided lesions were slowe and/or missed significantly more visual targets on the left side. In contrast, all patients with left hemispheric lesions, except one, were not impaired in the search task. This study substantiates the thesis that the right hemisphere has a dominant role in spatial attention mechanisms. (Fournal of Neurology Neurosurgery, and Psychiatry 2001;71:1-5)

\section{Visual symptoms of PEAS}

The Centers for Disease Control and Prevention have designated the human illness apparently associated with exposure to estuaries inhabited by toxin forming dinoflagellates as PEAS. This acute apparent neurotoxin mediated illness may manifest itself with nonspecific symptoms including cough, diarrhoea, headache, and fatigue. However, in a recent study from North Carolina it was noted that vision pattern detection and visual contrast sensitivity are sharply reduced in affected patients. This can be monitored objectively and is useful in diagnosing and monitoring treatment in PEAS. Visual contrast sensitivity improvements can be documented when the patients are treated. (Environmental Health Perspectives 2001;109:53945)

\section{Stroke after coronary artery bypass}

Early postoperative stroke is a serious adverse effect after coronary artery bypass grafting. It is associated with a high short term morbidity and mortality. In a recent study from Washington $\mathrm{DC}$ over a 10 year period more than 16000 patients underwent coronary artery bypass grafting. The risk for postoperative stroke was significantly increased in patients with chronic renal insufficiency, recent cardiomyofarction, previous cerebral vascular accident, carotid artery disease, hypertension, and diabetes. Postoperative stroke was associated with a longer postoperative stay. (Stroke 2001;32:1508-13)

The use of statins and the development of deep vein thrombosis

Some but not all of the benefit of statins for the prevention of cardiovascular disease is due to the antithrombotic properties. A recent study from Ontario, Canada, investigated whether the use of statins had any effect on deep vein thrombosis. In this study of patients over 65 years of age the use of statins was associated with a $22 \%$ relative risk reduction of developing deep vein thrombosis. The study did not evaluate the efficacy of statins for primary and secondary prevention of deep vein thrombosis. (Archives of Internal Medicine 2001;161:1405-10)

HIV resistance and a gene mutation

A report from New York University School of Medicine suggests that people who inherit only one copy of a mutated gene that has an effect on HIV's ability to enter CD4T helper cells appear to be substantially less likely to become infected with the virus. If this protective effect is proved in further studies it may lead to the use of drugs currently under development that are designed to mimic the mutations effect. The gene under study encodes a cell surface receptor called CCR5, which HIV-1 usually requires (in addition to the $\mathrm{CD} 4$ receptor) to invade CD4T cells. (fournal of Acquired Immune Deficiency Syndromes 2001;15:10-13) 